\title{
Xanomeline derivative EUK1001 attenuates Alzheimer's disease pathology in a triple transgenic mouse model
}

\author{
ZIYAN LI $^{1}$, KAILI JIA ${ }^{1}$, YALE DUAN ${ }^{2}$, DONG WANG $^{2}$, ZONGLI ZHOU $^{2}$ and SUZHEN DONG ${ }^{1}$ \\ ${ }^{1}$ Shanghai Engineering Research Center of Molecular Therapeutics and New Drug Development, School \\ of Chemistry and Molecular Engineering; ${ }^{2}$ Key Lab of Brain Functional Genomics, Shanghai Institute \\ of Brain Functional Genomics, East China Normal University, Shanghai 200062, P.R. China
}

Received February 7, 2017; Accepted September 5, 2017

DOI: $10.3892 / \mathrm{mmr} .2017 .7502$

\begin{abstract}
Agonists of M1 muscarinic acetylcholine receptors are promising therapeutic agents for the treatment of Alzheimer's disease (AD). An example of one of these agents is xanomeline, which has been a leading candidate, however induces various unwanted adverse effects. 3-[3-(3-florophenyl-2-propyn-1-ylthio)-1,2,5thiadiazol-4-yl]-1,2,5,6-tetrahydro-1-methylpyridine oxalate (EUK1001), a fluorinated derivative of xanomeline, has been demonstrated to attenuate $\mathrm{AD}$-like neurodegenerative pathology in presenilin-deficient mice, which has no $\beta$-amyloid (A $\beta$ ) pathology. The present study assessed the effect of EUK1001 on the behavioral performance of the 3xTg-AD model of AD. EUK1001 treatment decreased cognitive deficits in male and female AD mice in the Morris water maze test and novel object recognition tasks. EUK1001 also decreased A $\beta 42$, however not $\mathrm{A} \beta 40$ in the cortex and hippocampus of $\mathrm{AD}$ mice. EUK1001 may also alter amyloid precursor protein processing to a nonamyloidgenic pathway in vitro. These results demonstrate that EUK1001 may ameliorate the cognitive dysfunction of AD mice, possibly by reducing $\mathrm{A} \beta$ production. Therefore, EUK1001 may be an effective treatment for AD.
\end{abstract}

\section{Introduction}

Alzheimer's disease (AD) is the most common neurodegenerative disorder with progressive deterioration of memory in the elderly. There are nearly 40 million AD patients worldwide and prevalence is increasing because of increased longevity

Correspondence to: Dr Suzhen Dong, Shanghai Engineering Research Center of Molecular Therapeutics and New Drug Development, School of Chemistry and Molecular Engineering, East China Normal University, 3663 Zhongshan North Road, Shanghai 200062, P.R. China

E-mail: szdong@brain.ecnu.edu.cn

Key words: Alzheimer's disease, muscarinic receptor agonist, EUK1001, A $\beta 40$, triple transgenic mice, water maze, novel object recognition, neurodegeneration, nonamyloidgenic processing and the aging population. Therefore, it is critical to understand the underlying pathology of $\mathrm{AD}$ and to find promising therapies.

Beta amyloid $(A \beta)$ cascades, the posttranslational modification of tau proteins and cholinergic dysfunctions, are considered the most important hypotheses of AD pathology, but no theory alone is sufficient to explain the diversity of biochemical pathways and abnormalities leading to AD. Currently, the majority of FDA-approved therapies for AD are designed to restore cholinergic function. Inhibitors of acetylcholinesterase (AChE), for example, are an effective method to correct cholinergic deficiency in some AD cases (1). However, their effectiveness diminishes over time because of the progressive loss of cholinergic neurons. In contrast, it has been reported that levels of M1 muscarinic acetylcholine receptors (mAchRs) are well preserved in the brains of AD patients relative to other $\mathrm{mAch}$ Rs and nicotinic receptors (2). Therefore, M1 receptor agonists might be an alternative method of restoring cholinergic function for AD treatment. In support of this notion, it has been demonstrated that activation of M1 receptors can attenuate AD-related pathology including memory impairment, amyloid deposition, and tau phosphorylation (3-6). One agonist of the M1 receptor, xanomeline, has been a promising candidate for such treatment. Xanomeline has indeed been shown to induce dose-dependent improvement of cognitive function and behavioral performance in AD patients, but its clinical utility is limited owing to intolerable muscarinic adverse effects (3). In order to reduce the unwanted adverse effects of this agent, a derivative of xanomeline named EUK1001 has been developed, which showed higher affinity for M1 mAChRs and significantly less toxicity than xanomeline (7). Moreover, EUK1001 has been shown to improve cognitive function, enhance hippocampal synaptic plasticity in aged mice $(7,8)$, and to induce hippocampal neurogenesis in adult mice (9). Most importantly, EUK1001 can ameliorate recognition memory, attenuate brain degeneration, and suppress brain tau hyperphosphorylation in the presenilin-deficient mouse model (6), suggesting that EUK1001 might be a promising therapeutic agent for AD treatment. Although presenilin1/presenilin2 conditional double knockout mice (PS cDKO mice) show AD-like neurodegenerative phenotypes, these mice have decreased $A \beta$ levels in the brain (10-13), inconsistent with AD patients. Thus, these mice might not be a suitable animal model for testing anti-AD 
agents. Therefore, the effects of EUK1001 on neurodegeneration need to be further assessed in other animal models of AD.

It is well known that the triple transgenic mice expressing human Swedish double-mutated APP (APPsw), human mutated four-repeat tau (tauP301L), and human mutated presenilin1 (PS1M146V) (3xTg-AD mice) progressively develop A $\beta$ plaques and neurofibrillary tangles and are the best presently available AD animal model (14). In the present study, we explored the effects of EUK1001 on the cognitive phenotype of this 3xTg-AD mouse model and analyzed possible underlying mechanisms.

\section{Materials and methods}

Animals. Triple transgenic mice (3xTg-AD) harboring hAPPswe, htauP301L, and hPS1M146V transgenes were originally generated by Oddo et al (14). The transgenic mice used in this study were purchased from the colony maintained by the Jackson Laboratory and bred in our lab. Wild-type mice on the same strand background (B6129SF2/J) served as controls according to the instructions of the Jackson Laboratory. All mice were maintained in a specific-pathogen-free (SPF) facility on a 12 -h light/dark cycle $\left(20-26^{\circ} \mathrm{C}\right.$ and $40-70 \%$ humidity). All animal procedures were approved by the Committee for the Care and Use of Laboratory Animals at ECNU, China.

Compounds and treatment. Both xanomeline and EUK1001 (7) were synthesized in the laboratory of Dr. Xiaoping Lei at Peking University. Male and female 3xTg-AD mice were divided into different groups because previous studies have demonstrated that behavior performance of 3xTg-AD mice varies by sex with females performing worse than males (15). Six-month-old male 3xTg-AD mice were randomly divided into two groups (EUK1001 and control) and female mice into three groups (EUK1001, xanomeline, and control; $n=10-12$ per group). For each group, mice were injected intraperitoneally with xanomeline $(1 \mathrm{mg} / \mathrm{kg})$, EUK1001 (1 mg/kg), or saline (as the vehicle control) daily for three months. Male and female wild-type mice were also divided into two groups (EUK1001 and control; $n=10-12$ per group) and were administered EUK1001 or saline identically to the 3xTg-AD mice. These compounds were also administered during the two weeks of behavioral testing.

Water maze. We used a circular pool (1.2 $\mathrm{m}$ in diameter) with a platform placed in the center of any quadrant, submerged $1 \mathrm{~cm}$ beneath the water surface. The maze was located in a room containing several simple extra-maze visual cues. The training protocol consisted of four sessions, with one session (four trials) per day. The starting quadrant was randomly assigned for each animal but each session (four trials) included all four starting quadrants. To reduce stress, in both the hidden and cued versions of the task, mice were placed on the platform for $10 \mathrm{sec}$ prior to the first training trial. Mice were allowed to find and escape onto the submerged platform. If a mouse failed to find the platform within $60 \mathrm{sec}$, we manually guided it to the platform and allowed to remain there for $30 \mathrm{sec}$. After this, each mouse was placed into a holding cage under a warming lamp for $25 \mathrm{sec}$ until the next trial. During the transfer test, the platform was removed and the mice were allowed to swim in the pool for $60 \mathrm{sec}$. We recorded the time spent in each quadrant by each mouse and the time until each mouse crossed the platform after searching.

Novel objectrecognition. This task was conducted as previously described (16). Briefly, mice were extensively handled and then individually habituated to an open field box $(50 \times 50 \times 25 \mathrm{~cm})$ for 15 min every day for 3 days. During each training session, each mouse was allowed to explore freely for $15 \mathrm{~min}$ in the open field box with two novel objects placed in the center. In the 1-h and 24-h retention tests, mice were placed back into the same box for $15 \mathrm{~min}$, and one of the objects during the training session was replaced with a novel object. The discrimination index is calculated as the ratio of the time spent exploring the novel object divided by the total exploration time for both objects, and is used to evaluate recognition memory.

A $\beta$ ELISA. A $\beta$ measurements were performed by the Invitrogen Human $A \beta 40$ and $A \beta 42$ ELISA kit (Life Technologies, New York, NY, USA) following the manufacturer's protocols. Cortex or hippocampus samples were sonicated $(5 \mathrm{sec})$ in four volume protein lysis buffer containing protease inhibitors. The lysate was centrifuged $\left(18,000 \mathrm{xg}\right.$, for $10 \mathrm{~min}$ at $\left.4^{\circ} \mathrm{C}\right)$. The supernatant was considered soluble and pellets were further extracted by sonicating with formic acid and further centrifugation. Prior to the ELISA, insoluble samples were diluted 3 times with Tris $1 \mathrm{M}, \mathrm{pH} 10$, and soluble samples were diluted 5 times in ELISA buffer. Results are expressed as pg/mg of protein measured by the Bradford method, using BSA as standard.

Plasmid construction. The whole coding sequence (CDS) of the human muscarinic M1 receptor (about $1.4 \mathrm{~kb}$, accession no. NM_000738) was obtained by PCR and inserted into the HindIII and EcoRI sites of pcDNA3.1(+). The whole CDS of human APP Swedish mutant (APPsw) (about $2.1 \mathrm{~kb}$, accession no. NM_201414) was obtained by PCR using brain cDNA of 3xTg-AD mice. The PCR product was digested by restricted enzymes HindIII and Xba I and subcloned into pcDNA3.1(+). The positive clones of pcDNA3.1-hM1 (hM1) and pcDNA3.1-hAPPsw (hAPPsw) were validated by sequencing and their expression was tested in N2a cells after transfection by western blotting.

Cell culture and treatment. N2a cells were cultured in Dulbecco's Modified Eagle's Medium (high glucose with L-glutamine; Gibco; Thermo Fisher Scientific, Inc., Waltham, MA, USA) supplemented with $10 \%$ Fetal Bovine Serum (FBS; PAA Laboratories Inc., Dartmouth, MA, USA), and $1 \%$ penicillin/streptomycin (PAA Laboratories Inc.), $\mathrm{pH} 7.2$, in a humidified $5 \% \mathrm{CO}_{2}$ water-jacketed incubator (Thermo Forma Inc., Waltham, MA, USA). To analyze the effects of M1 receptor activation on APP processing in N2a cells, N2a cells were cotransfected with the following combined plasmids: (1) hAPPsw/hM1, (2) hAPPsw/pc (pcDNA3.1), (3) pc/hM1, (4) pc/pc, using Lipofectiome 2000 according to the manufacturer's protocol. Forty-eight h after transfection, N2a cells were rinsed twice with FBS-free Opti-MEM, and treated for $6 \mathrm{~h}$ with or without xanomeline or EUK1001 in Opti-MEM.

Western blot analysis. After xanomeline or EUK1001 treatment, the cotransfected cells and their medium were collected. 
The medium was centrifuged for $5 \mathrm{~min}$ at $14,000 \mathrm{x}$ at $4^{\circ} \mathrm{C}$ and the supernatant was subjected to western blotting after the addition of BSA (final concentration: $10 \mu \mathrm{g} / \mathrm{ml}$ ), which served as a loading control. The treated cotransfected cells were lysed on ice using a lysis buffer (Beyotime, Shanghai, China) containing $1 \mathrm{mM}$ PMSF, followed by centrifugation at $14,000 \mathrm{x} \mathrm{g}$ at $4^{\circ} \mathrm{C}$ for $5 \mathrm{~min}$. Protein concentrations were determined using a BCA kit (Beyotime). Equal amounts of protein were resolved by SDS-PAGE and electrically transferred onto nitrocellulose membranes (Millipore, Billerica, MA, USA). After blocking for $1 \mathrm{~h}$ with $5 \%$ non-fat milk at room temperature, the blots were incubated with the primary antibodies for $1 \mathrm{~h}$, and then with the secondary antibodies for $1 \mathrm{~h}$ at room temperature. Proteins were visualized with an ECL kit (CWBio, Beijing, China). A Quantity One GelDoc XR gel imaging system (Bio-Rad Laboratories, Hercules, CA, USA) was used for detection and analysis of band intensity. Relative protein levels were normalized to BSA or $\beta$-actin. Antibodies used were: anti- $\beta$-Amyloid (DE2B4) (1:500; Santa Cruz Biotechnology, Santa Cruz, CA, USA), anti-Amyloid $\beta$-Precursor Protein (LN27) (1:1,000; Invitrogen), anti-BSA (1:500; Bioss, Beijing, China), $\beta$-actin (1:1,000; Cell Signaling Technology, Beverly, MA, USA) and proper HRP-conjugated secondary antibodies (1:5,000; KangChen Bio-tech, Shanghai, China).

Statistical analysis. Results, presented as mean \pm SEM, were analyzed by two way ANOVA and post-hoc Holm-Sidak method using Graphpad Prism. Student's t-test or Mann-Whitney U test was used for direct comparison of two groups using Sigmaplot 10.0 or SPSS 10.0 software.

\section{Results}

Effect of EUK1001 on cognitive function. To evaluate the therapeutic effects of the xanomeline derivative, EUK1001, on the cognitive phenotype of $\mathrm{AD}$, six-month-old homozygous 3xTg-AD and wild-type mice were administered EUK1001, xanomeline, or saline daily for three months. Based on previous results of in vivo pharmacological and behavioral tests, we tested the doses of $1 \mathrm{mg} / \mathrm{kg}$ xanomeline and EUK1001 (7). We evaluated the effects of these drugs on learning and memory using two different behavioral paradigms: Morris water maze and novel object recognition. Both tasks are dependent on the the hippocampus, a site where the $A \beta$ and tangle pathology is most severe in 3xTg-AD mice (14). We discussed the results of these tests in turn.

In the Morris water maze task, both male and female saline-treated AD mice (ct-ad-m and ct-ad-f) exhibited greater escape latencies than saline-treated wild-type mice (Fig. 1A and $\mathrm{B} ; \mathrm{P}<0.01)$. Both male and female EUK1001-treated mice performed as well as control mice (Fig. $1 \mathrm{~A}$ and $\mathrm{B} ; \mathrm{P}>0.05$ ). Xanomeline-treated female AD mice spent less time to find the platform compared to saline-treated AD mice (Fig. 1A; $\mathrm{P}<0.05)$. Similarly, in the probe trial, time spent in the target quadrant was significantly shorter for ct-ad-m and ct-ad-f mice compared to wild type (Fig. $1 \mathrm{C}$ and $\mathrm{D} ; \mathrm{P}<0.05$ ), while this time was not significantly different between wild-type and EUK1001-treated mice (Fig. 1C and D; P>0.05). Xanomeline treatment also ameliorated the performance of $\mathrm{X}$-ad-f mice in the probe trial (Fig. 1D; $\mathrm{P}<0.05$ ). We found no significant difference between xanomeline-treated female mice and EUK1001-treated female mice (Fig. 1D; P>0.05). These results suggest that EUK1001 treatment can rescue impairment of spatial memory in AD mice although it has no evident effect on the performance of wild-type mice.

In the novel object recognition task, we found no preference between the two objects in any groups during the training sessions. However, both the ct-ad-m and ct- ad-f mice spent less time exploring the novel object during both 1 and 24-h retention tests than wild-type controls (Fig. 2A and $\mathrm{B} ; \mathrm{P}<0.05$ ). The EUK1001-treated AD mice showed significantly more exploring of the novel object than control AD mice (Fig. 2A and $\mathrm{B} ; \mathrm{P}<0.05)$. Xanomeline-treated female mice performed similarly to control AD mice (Fig. 2A and B; P>0.05). EUK1001 treatment did not induced any significant changes to exploring preference in wild type mice (Fig. 2A and B; $P>0.05)$. These results suggest that EUK1001 treatment also enhanced recognition memory of AD mice.

Effect of EUK1001 on A $\beta$ level in the brain. All mice were sacrificed $24 \mathrm{~h}$ after the final novel object recognition trial, and their brains were isolated and processed for biochemical evaluation. To determine the effects of EUK1001 administration on the AD neuropathology present in the 3xTg-AD mice, we compared the $A \beta 40$ and $A \beta 42$ levels in the cortices and hippocampi of saline- and EUK1001-treated AD mice using sandwich ELISA. The results showed that EUK1001 treatment led to a significant decrease of A $\beta 42$ level in both the hippocampi and cortices of AD mice (Fig. $3 \mathrm{~A} ; \mathrm{P}<0.01$ ), but had no significant effect on A 340 level (Fig. 3B; P>0.05).

Effect of EUK1001 on APP processing in vitro. It is well known that APP undergo sequential proteolytic processing by two pathways: The $\alpha$ pathway and the $\beta$ pathway. If APP is cleaved by $\alpha$-secretase, which produces sAPP $\alpha$, the pathway is non-anyloidogenic. So we wondered whether the EUK1001 treatment had an effect on $A \beta 42$ levels via altered APP processing to $\alpha$ pathway. In this regard, APPsw and M1 receptor-overexpressing $\mathrm{N} 2 \mathrm{a}$ cell models were established for the analysis of sAPP $\alpha$ (alpha-secretase-cleaved APP) secretion (The overexpression was validated by real-time PCR experiments, Figs. 4A and B). Cell media was collected for detection of sAPP $\alpha$ by western blotting using DE2B4 antibody, which recognizes SAPP $\alpha$ and amino acids 1-17 of A $\beta$. Cell lysate was used to detect the expression of APPsw using the LN27 antibody which recognizes the N-terminus of APP. Results indicated that xanomeline and EUK1001 both increased sAPP $\alpha$ levels in the media of N2a cell cotransfected with APPsw and M1 without affecting total expression levels of APPsw (Fig. 4C-E; $\mathrm{P}<0.01)$. Conversely, xanomeline and EUK1001 had no effect on the secretion of SAPP $\alpha$ in APPsw/pcDNA3.1 cotransfected N2a cells (Fig. 4F-H; P>0.05), which suggests that the effect of xanomeline and EUK1001 was dependent on the M1 receptor.

\section{Discussion}

Activation of muscarinic receptors by M1 agonists including xanomeline has been demonstrated to be an effective therapeutic strategy for AD. However, their clinic utility has so 

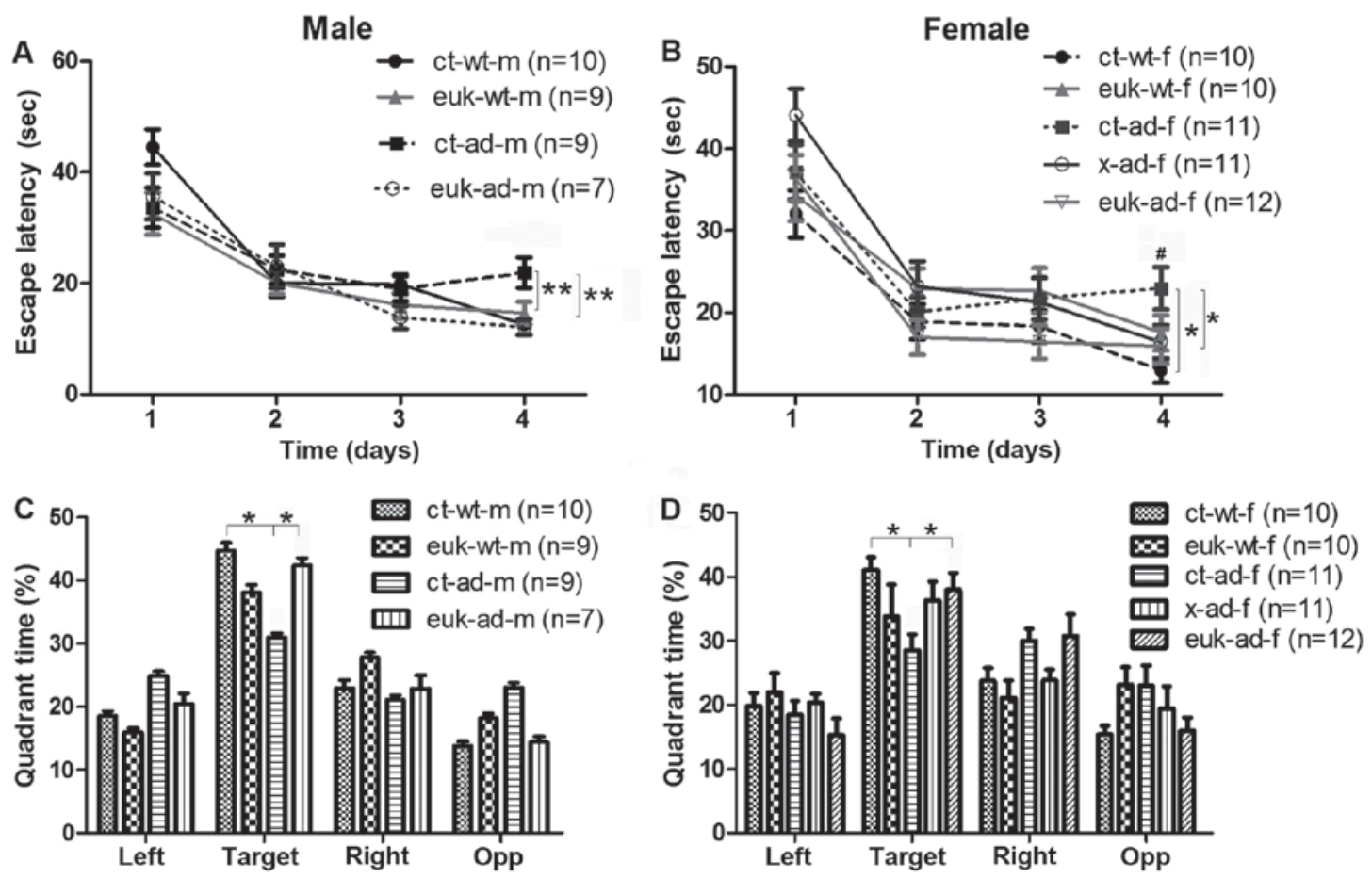

Figure 1. EUK1001 treatment rescued the spatial memory deficits of 3xTg-AD mice. (A and B) During training, both male (A) and female (B) AD mice exhibited a greater latency than wild-type mice. In contrast, EUK1001-treated AD mice had a similar latency to wild-type. (C and D) During the probe session, both male (C) and female (D) AD mice spent less time in the target quadrant than wild-type mice, while EUK1001-treated AD mice spent a similar duration of time in the target quadrant as wild-type mice. ${ }^{* *} \mathrm{P}<0.01,{ }^{*} \mathrm{P}<0.05$ : $c t$ - $a d$ vs. $c t$-wt; euk-ad vs. $c t$ - $a d .{ }^{~} \mathrm{P}<0.05$ : $X$ - $a d$ vs. ct-ad. $\mathrm{x}$, xanomeline; euk, EUK1001; ct, saline; ad, Alzheimer's disease; m, male; f, female; wt, wild-type;

A

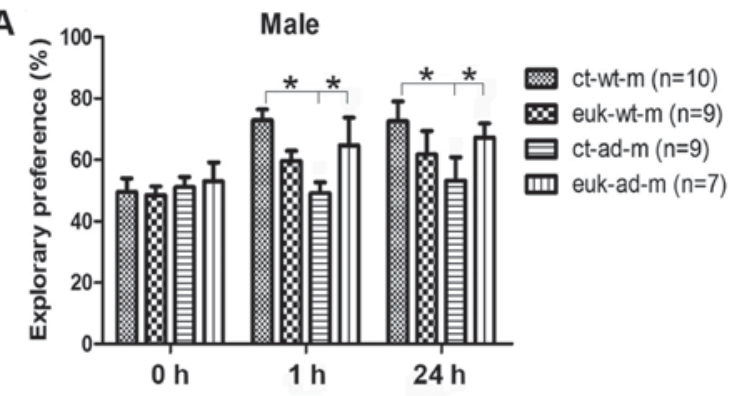

B

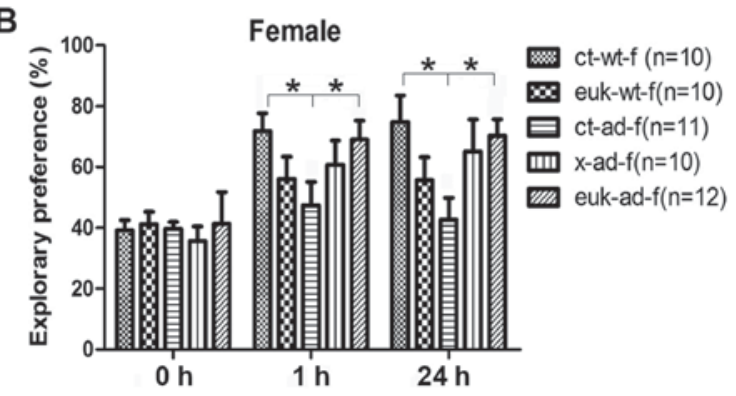

Figure 2. EUK1001 treatment rescued the recognition memory impairment of 3xTg-AD mice. (A and B): Both male (A) and female (B) AD mice showed no exploration preference to the novel objects in both the 1 and 24-h retention trials. However, EUK1001-treated AD mice displayed significant preference for the novel object, similar to wild-type mice. ${ }^{*} \mathrm{P}<0.05$ : $c t$-ad vs. $c t$-wt; euk-ad vs. $c t$-ad. x, xanomeline; euk, EUK1001; ct, saline; ad, Alzheimer's disease; m, male; f, female; wt, wild-type.

far been limited owing to severe adverse effects (3). The derivative of xanomeline, EUK1001, has lower toxicity and in previous research has been shown to attenuate AD-like neurodegeneration in presenilin-deficient mice (6). The mice, however, do not mimic all of the hallmarks of AD patients without $A \beta$ production (12). The present study provides evidence for the beneficial effects of EUK1001 for AD pathology using the well-known 3xTg-AD mouse model. Our results indicate that three-month treatment with EUK1001 can ameliorate the impairment of spatial memory and recognition memory in both male and female 3xTg-AD mice. This is consistent with previous studies showing that selective M1 receptor agonist, AF267B, rescues cognitive deficits in 3xTg-AD mice (4).

Additionally, we have found that EUK1001 treatment led to a decrease of A $\beta 42$ level in AD mice and induced an increase of sAPP $\alpha$ production in N2a cells, suggesting that activation of M1 receptors by EUK1001 might alter APP processing to an alternative nonamyloidgenic pathway, which will be confirmed in our ongoing work. A similar effect was found in previous experiments (4). These results also demonstrated that the effect of M1 receptor activation on APP processing is mediated by the activation of PKC and ERK1/2 (4,17). Therefore, EUK1001 administration might enhance cognitive function of $3 \times \mathrm{Tg}-\mathrm{AD}$ mice by activating M1 mAChRs, which leads to the generation of $\alpha$-secretase-generated products through activation of PKC and ERK1/2. Further study will be done to elucidate whether the effect of M1 receptor activation on APP processing is mediated by the activation of PKC and ERK1/2 pathway M1 mAchRs represent a viable target for AD treatment, and highly selective allosteric agonists of M1 receptors are promising compounds for AD therapies (18). The principal challenge has been to find selective compounds that activate the M1 receptor subtype without also activating M2 or M3 receptors, which are associated with undesirable adverse effects. All previous 

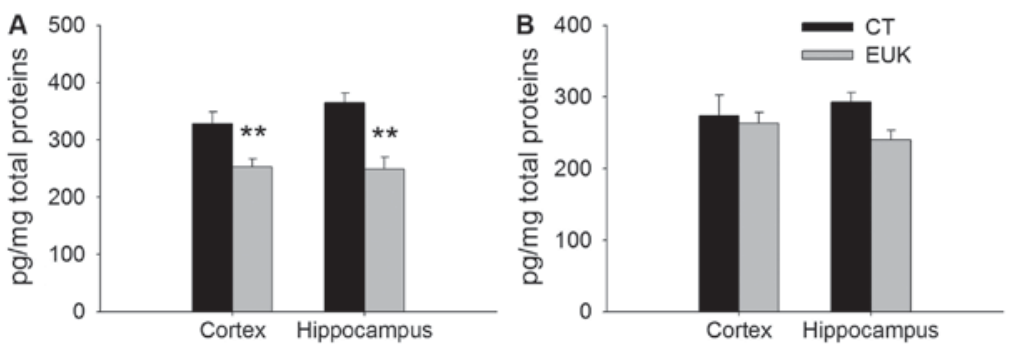

Figure 3. EUK1001 administration decreased A $\beta 42$ level but not $A \beta 40$ in the cortex and hippocampus. (A) A $\beta 42$ level; (B) $A \beta 40$ level. $A \beta$ level was detected using an $\mathrm{A} \beta$ Elisa kit. ${ }^{* *} \mathrm{P}<0.01$. euk, EUK1001; ct, saline.

A

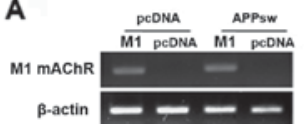

C

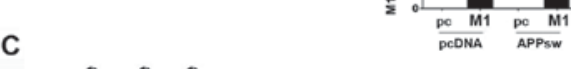

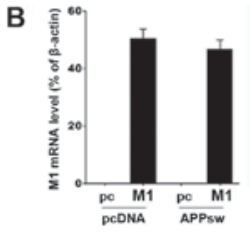

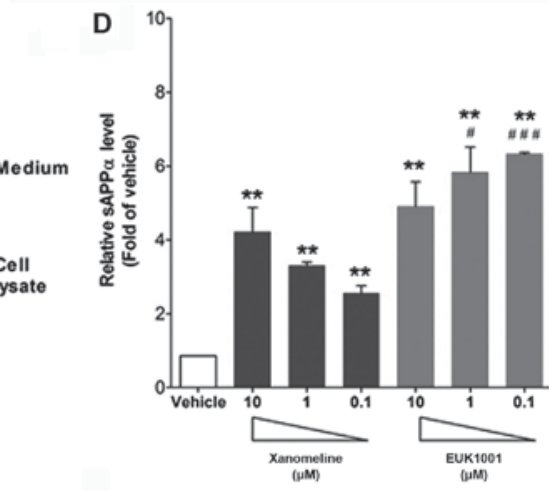

E

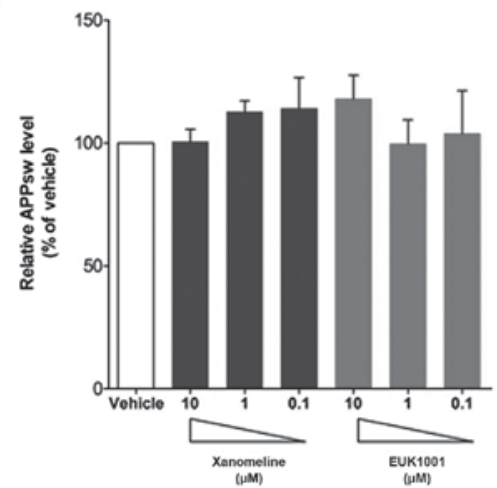

G

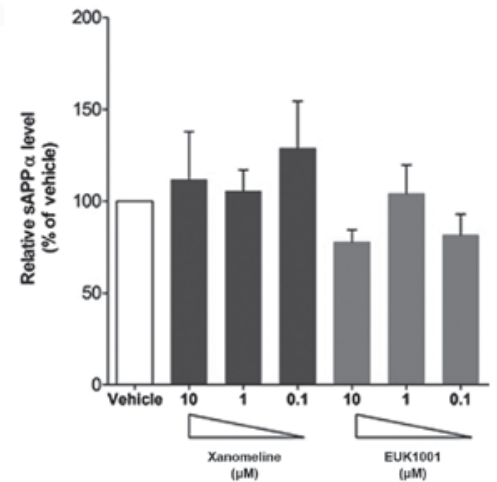

$\mathbf{F}$

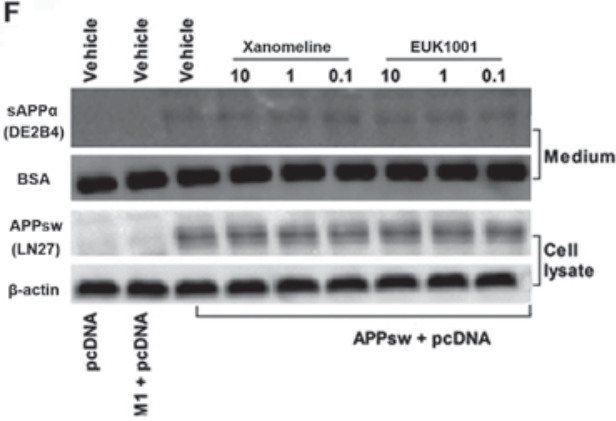

H

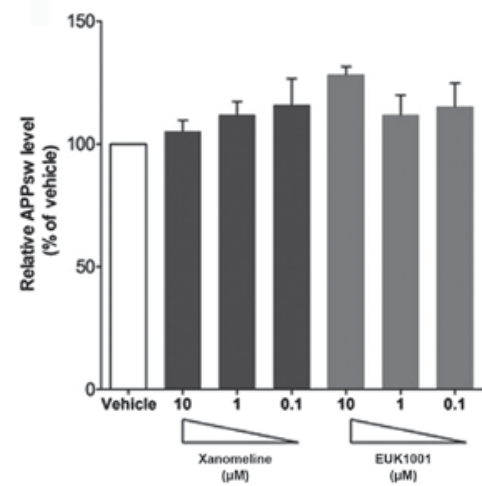

Figure 4. EUK1001 treatment increased sAPP $\alpha$ by activating M1 receptors in transfected N2a cells. N2a cells were transiently cotransfected with hAPPsw and M1 receptors or hAPPsw and pcDNA3.1. Potent M1 receptor expression was observed in APPsw/M1-N2a and pc/M1-N2a cells, but not in APPsw/pc-N2a and pc/N2a cells, as revealed by (A) PCR and (B) reverse transcription-qPCR. Then the cells were treated with or without xanomeline or EUK1001 in Opti-MEM. Proteins in cell medium and lysate were collected for western blotting. For the detection of SAPPa in the medium, BSA was added and served as a loading control. Relative protein levels were normalized to $\beta$-actin (BSA for sAPP $\alpha$ ) and are shown +SEM. (C and D) Xanomeline and EUK1001 effectively increased sAPP $\alpha$ levels in the medium, (C and E) without affecting the total expression levels of APPsw. (F-H) Xanomeline and EUK1001 had no effect on secreted sAPPa levels or total APPsw level in hAPPsw/pcDNA3.1 cotransfected N2a cells. ${ }^{* *} \mathrm{P}<0.01$, vs. vehicle; ${ }^{~} \mathrm{P}<0.05,{ }^{\text {,\#t }} \mathrm{P}<0.001$, vs. Xanomeline at the same concentration. hAPPsw, human Swedish double-mutated amyloid precursor protein; sAPP, soluble amyloid precursor protein; mAchR, muscarinic acetylcholine receptor; BSA, bovine serum albumin; PCR, polymerase chain reaction. 
compounds have been discontinued owing to inadequate selectivity. Therefore, the suitability of EUK1001 as a therapy for AD lies in its specificity. Studies in our lab have indicated that the effects of EUK1001 on hippocampal long-term potentiation are blocked by pirenzepine, a selective M1 antagonist, but not by the selective M2/M4 antagonist methoctramine (7). This suggests that EUK1001 was able to affect hippocampal long-term potentiation via M1 receptor stimulation. Moreover, EUK1001 has been shown to have low toxicity in mice (7). Thus, EUK1001 remains a promising therapeutic agent for the treatment of $\mathrm{AD}$. However, these effects and possible adverse effects still require further investigation before and during clinical application.

Currently, a fully selective muscarinic M1 receptor agonist, HTL9936, is undergoing Phase 1 clinical trials in order to develop a new medicine for improving cognitive function in patients with AD (19). The present paper has identified another agent, EUK1001, which could alleviate memory impairment by decreasing $A \beta$ pathology. This drug also attenuated the neurodegenerative phenotypes in another AD-like rodent model (6). Thus, future work should be performed to investigate the potential role of EUK1001 for AD therapy.

\section{Acknowledgements}

The present study was supported by the Natural Science Foundation of Shanghai, no. 16ZR1410500. We thank the English native speaker in Editage for professional language editing of the manuscript.

\section{References}

1. Knapp MJ, Knopman DS, Solomon PR, Pendlebury WW Davis CS and Gracon SI: A 30-week randomized controlled trial of high-dose tacrine in patients with Alzheimer's disease. The Tacrine Study Group. JAMA 271: 985-991, 1994.

2. Nordberg A, Alafuzoff I and Winblad B: Nicotinic and muscarinic subtypes in the human brain: Changes with aging and dementia. J Neurosci Res 31: 103-111, 1992.

3. Bodick NC, Offen WW, Levey AI, Cutler NR, Gauthier SG, Satlin A, Shannon HE, Tollefson GD, Rasmussen K, Bymaster FP, et al: Effects of xanomeline, a selective muscarinic receptor agonist, on cognitive function and behavioral symptoms in Alzheimer disease. Arch Neurol 54: 465-473, 1997.

4. Caccamo A, Oddo S, Billings LM, Green KN, Martinez-Coria H, Fisher A and LaFerla FM: M1 receptors play a central role in modulating AD-like pathology in transgenic mice. Neuron 49: 671-682, 2006.

5. Fisher A: M1 muscarinic agonists target major hallmarks of Alzheimer's disease-an update. Curr Alzheimer Res 4: 577-580, 2007.
6. Wang D, Yang L, Su J, Niu Y, Lei X, Xiong J, Cao X, Hu Y, Mei B and Hu JF: Attenuation of neurodegenerative phenotypes in Alzheimer-like presenilin 1/presenilin 2 conditional double knockout mice by EUK1001, a promising derivative of xanomeline. Biochem Biophys Res Commun 410: 229-234, 2011.

7. Cui Y, Wang D, Si W, Lv W, Niu Y, Lei X, Hu Y and Cao X: Enhancement of memory function in aged mice by a novel derivative of xanomeline. Cell Res 18: 1151-1153, 2008.

8. Cui YH, Si W, Yin L, An SM, Jin J, Deng SN and Cao XH: A novel derivative of xanomeline improved memory function in aged mice. Neurosci Bull 24: 251-257, 2008.

9. Zhang XL, Gong Q, Zhang S, Wang L, Hu Y, Shen H and Dong S: 3-[3-(3-florophenyl-2-propyn-1-ylthio)-1,2, 5-thiadiazol-4-yl]-1,2,5,6-tetrahydro-1-methylpyridine oxalate, a novel xanomeline derivative, improves neural cells proliferation and survival in adult mice. Neural Regen Res 7: 24-30, 2012.

10. Beglopoulos V, Sun X, Saura CA, Lemere CA, Kim RD and Shen J: Reduced beta-amyloid production and increased inflammatory responses in presenilin conditional knock-out mice. J Biol Chem 279: 46907-46914, 2004.

11. Feng R, Wang H, Wang J, Shrom D, Zeng X and Tsien JZ: Forebrain degeneration and ventricle enlargement caused by double knockout of Alzheimer's presenilin-1 and presenilin-2. Proc Natl Acad Sci USA 101: 8162-8167, 2004.

12. Saura CA, Choi SY, Beglopoulos V, Malkani S, Zhang D, Shankaranarayana Rao BS, Chattarji S, Kelleher RJ III, Kandel ER, Duff K, et al: Loss of presenilin function causes impairments of memory and synaptic plasticity followed by age-dependent neurodegeneration. Neuron 42: 23-36, 2004.

13. Jiang X, Zhang D, Shi J, Chen Y, Zhang P and Mei B: Increased inflammatory response both in brain and in periphery in presenilin 1 and presenilin 2 conditional double knock-out mice. J Alzheimers Dis 18: 515-523, 2009.

14. Oddo S, Caccamo A, Shepherd JD, Murphy MP, Golde TE, Kayed R, Metherate R, Mattson MP, Akbari Y and LaFerla FM: Triple-transgenic model of Alzheimer's disease with plaques and tangles: Intracellular Abeta and synaptic dysfunction. Neuron 39: 409-421, 2003

15. Clinton LK, Billings LM, Green KN, Caccamo A, Ngo J, Oddo S, McGaugh JL and LaFerla FM: Age-dependent sexual dimorphism in cognition and stress response in the 3xTg-AD mice. Neurobiol Dis 28: 76-82, 2007.

16. Dong S, Li C, Wu P, Tsien JZ and Hu Y: Environment enrichment rescues the neurodegenerative phenotypes in presenilinsdeficient mice. Eur J Neurosci 26: 101-112, 2007.

17. Haring R, Fisher A, Marciano D, Pittel Z, Kloog Y, Zuckerman A, Eshhar $\mathrm{N}$ and Heldman E: Mitogen-activated protein kinase-dependent and protein kinase $\mathrm{C}$-dependent pathways link the $\mathrm{m} 1 \mathrm{muscarinic}$ receptor to beta-amyloid precursor protein secretion. J Neurochem 71: 2094-2103, 1998.

18. Caccamo A, Fisher A and LaFerla FM: M1 agonists as a potential disease-modifying therapy for Alzheimer's disease. Curr Alzheimer Res 6: 112-117, 2009.

19. HTL-9936 is a selective muscarinic M1 agonist designed to improve cognitive function in patients with $\mathrm{AD}$ and other diseases. http://newdrugapprovals.org/2014/05/05/htl-9936-is-a-selectivemuscarinic-m1-agonist-designed-to-improve-cognitive-functionin-patients-with-ad-and-other-diseases/ 